\title{
Detection of X-ray-Emitting Hypernova Remnants in M101
}

\author{
Q. Daniel Wang \\ Dearborn Observatory, Northwestern University \\ 2131 Sheridan Road, Evanston, IL 60208-2900 \\ Electronic mail: wqd@nwu.edu
}

\begin{abstract}
Based on an ultra deep (230 ks) ROSAT HRI imaging of M101, we have detected 5 X-ray sources that coincide spatially with optical emission line features previously classified as supernova remnants in this nearby galaxy. Two of these coincidences (SNR MF83 and NGC5471B) most likely represent the true physical association of X-ray emission with shock-heated interstellar gas. MF83, with a radius of $\sim 134 \mathrm{pc}$, is one of the largest remnants known. NGC5471B, with a radius of $30 \mathrm{pc}$ and a velocity of at least $350 \mathrm{~km} \mathrm{~s}^{-1}$ (FWZI), is extremely bright in both radio and optical. The X-ray luminosities of these two shell-like remnants are $\sim 1$ and $3 \times 10^{38} \mathrm{ergs} \mathrm{s}^{-1}(0.5-2 \mathrm{keV})$, about an order of magnitude brighter than the brightest supernova remnants known in our Galaxy and in the Magellanic Clouds. The inferred blastwave energy is $\sim 3 \times 10^{52} \mathrm{ergs}$ for NGC5471B and $\sim 3 \times 10^{53} \mathrm{ergs}$ for MF83. Therefore, the remnants likely originate in hypernovae, which are a factor of $\gtrsim 10$ more energetic than canonical supernovae and are postulated as being responsible for $\gamma$-ray bursts observed at cosmological distances. The study of such hypernova remnants in nearby galaxies has the potential to provide important constraints on the progenitor type, rate, energetics, and beaming effect of $\gamma$-ray bursts.
\end{abstract}

Subject headings: galaxies: general — galaxies: individual (M101) - ISM: supernova remnants — X-rays: general — gamma-ray: bursts

\section{Introduction}

The face-on grand-design spiral M101 (Fig. 1) is an ideal site for studying various X-ray source populations and their relationship to other galactic components. At a well-determined distance $D=7.2 \pm 0.4 \mathrm{Mpc}$ (thus $1^{\prime}=2.1 \mathrm{kpc}$; Stetson et al. 1998), the galaxy is probably the largest in the sky, except for members of our Local Group. 
The galaxy is also in a direction of exceptionally low foreground Galactic extinction $\left[E(B-V) \sim 0 ; N_{H} \sim 1.1 \times 10^{20} \mathrm{~cm}^{-2}\right]$. A recent optical survey of the galaxy by Matonick \& Fesen (1997, MF hereafter) has identified 93 supernova remnant (SNR) candidates. The galaxy also contains numerous giant HII regions (GHRs) and complexes (GHCs), located primarily in outer spiral arms. Some of these GHRs are substantially brighter than the LMC 30 Dor nebula, which is the brightest HII region in the Local Group. Thus, M101 provides a particularly favorable circumstance to explore starburst-related phenomena.

Based on an ultra-deep image from the ROSAT High Resolution Imager (RHRI), Wang et al. (1999) have detected 51 sources with X-ray luminosities in the range of 0.4 - $20 \times 10^{38} \operatorname{ergs~s}^{-1}(0.5-2 \mathrm{keV})$. A cross-correlation of these X-ray sources with SNR candidates in the galaxy leads to a surprising discovery of 5 position coincidences. Unlike several previously-known X-ray-bright SNRs (in other nearby galaxies), which result from strong interaction between SN ejecta with dense circumstellar media, these apparently X-ray-emitting remnants in M101 are optically-resolved, and are thus relatively well evolved. Most importantly, the observed X-ray emission, if arising in shock-heated interstellar gas, implies the blastwave energy of each remnant is a factor of $\gtrsim 10$ greater than the energy ( 10 ${ }^{51}$ ergs; e.g., Hughes et al. 1998) expected for an SN. Therefore, the remnants may well be the products of hypernovae, as have been proposed to explain $\gamma$-ray bursts (GRBs) and their afterglows (Paczyński 1998; Fryer \& Woosley 1998). In this Letter, we describe this discovery and explore implications of such remnants in the study of GRBs. The term "hypernova", proposed first by Paczyński (1998) for a super-energetic GRB/afterglow event, is loosely used here to refer an explosion that appears too energetic to be a nominal SN.

\section{Cross-Correlation between X-ray Sources and SNR Candidates}

Table 1 lists 5 position coincidences between X-ray sources and known SNR candidates in M101 (see also Fig. 1). Except for NGC5461B, which was discovered in radio continuum (Skillman 1985; Yang, Skillman, \& Sramek 1994) and was confirmed in optical (Chu \& Kennicutt 1986; Chen \& Chu 1999), all other SNRs used in the cross-correlation are from the MF survey, which consists of both optical imaging and spectroscopic observations. The imaging observations covered five partially-overlapped regions of 8'7 $\times 8$ '.7 each. Emission nebulae with $[\mathrm{S} \mathrm{II}] / \mathrm{H} \alpha \gtrsim 0.45$ were identified as SNRs. Spectroscopic followup observations were carried out for 14 objects, including MF83 in Table 1. The SNR positions (J2000; Table 1) are accurate to $1^{\prime \prime}$, radii $R$ to about $\pm 5 \mathrm{pc}$, and both $\mathrm{H} \alpha$ Luminosities $L\left(H_{\alpha}\right)$ and $[\mathrm{S} \mathrm{II}] / \mathrm{H} \alpha$ ratios to $15 \%(\mathrm{MF})$. The $51 \mathrm{X}$-ray sources were detected with signal-to-noise ratios $>3.5$ within a field of $17^{\prime}$ radius covered by the RHRI image (Wang et al. 1999). 
The RHRI was sensitive to photons in the 0.1-2 keV range and had a point spread function (PSF) with a half power-encircled radius ranging from $\sim 2$ ".5 on-axis to $20^{\prime \prime}$ at $17^{\prime}$ off-axis. We corrected the astrometry of the image by matching centroids of 7 point-like X-ray sources with their optical counterparts. Table 1 includes only the SNRs whose position offsets $\Delta_{x-o}$ from X-ray source centroids are less than twice the $1 \sigma$ uncertainty radii $r_{\text {err }}$ (Wang et al. 1999). Table 1 also includes the projected galactocentric radius $R_{g}$, expanding velocity $v_{e}$, and optical morphology as well as the X-ray source number and $0.5-2 \mathrm{keV}$ luminosity of each remnant.

What is the probability for a chance superposition? The MF survey had a complete coverage of the central field of $R_{g} \lesssim 8^{\prime}$ from the M101 nucleus, which is at R.A., Dec. $(\mathrm{J} 2000)=54^{h} 3^{m} 12^{\mathrm{s}} .7,54^{\circ} 20^{\prime} 54^{\prime \prime}$ (Israel, et al. 1975). This field includes $29 \mathrm{X}$-ray sources and 87 SNRs. Our adopted $2 \sigma$ matching radii enclose a total region of $583 \operatorname{arcsec}^{2}$. The statistical probability to find by chance 1 match in the region, for example, is 0.45 . Four SNRs in Table 1 are within the field. It is quite possible that one or two of the coincidences in Table 1 are just the chance superposition. Indeed, H19 shows strong time variability and has a relatively hard spectrum (Wang et al. 1999). Thus the source most likely represents an accreting X-ray binary system. Although this system may still be related to the explosion responsible for the remnant, the X-ray emission is not due to shock-heated interstellar gas. To find 4 random SNR/X-ray source superpositions in the field, however, the probability falls to $\sim 10^{-3}$. Thus, we expect that at least some of the coincidences in Table 1 do represent physical associations between the X-ray sources and SNRs.

The most likely true X-ray-bright remnants in Table 1 are MF83 and NGC5471B. Their $\Delta_{x-o}$ are the smallest, well within the $1 \sigma$ error radii. Both of them are shell-like (the other three have filled morphology; Table 1). Most importantly, these two SNRs are known to be the most unusual in M101 (MF; Yang, Skillman, \& Sramek 1994; Chen \& Chu 1999). MF83 is one of the largest SNRs known. Its high [S II]/H $\alpha$ ratio and bright [OIII] emission clearly suggest that the optical nebula is shock heated; the $[\mathrm{O} \mathrm{III}] / \mathrm{H}_{\beta}(=2.0)$ ratio indicates a shock velocity of $\sim 86 \mathrm{~km} \mathrm{~s}^{-1}$ (Table 17 in MF). The remnant, located in a region just off a spiral arm, shows no evidence for an association with any concentration of OB stars. NGC5471B is an extended, nonthermal radio source with a flux of $4.4 \pm 0.8 \mathrm{mJy}$ at $30 \mathrm{~cm}$ and a spectral slope of -0.73 (Yang, Skillman, \& Sramek 1994). Based on optical echelle spectroscopy, Chu \& Kennicutt (1986) detected shocked gas with velocity $\gtrsim 350 \mathrm{~km} \mathrm{~s}^{-1}$ (FWZI) and inferred the required supernova blastwave energy as $\sim 10^{52}$ ergs. Therefore, the identification of both remnants are firm and they all appear to be unusually energetic. 


\section{Constraints on Remnant Parameters}

To characterize the spectral properties of X-ray-emitting plasma, we have further analyzed a ROSAT PSPC observation of M101 (Wang et al. 1999). Though only with a limited spatial resolution of $\sim 30^{\prime \prime}$ FWHM on-axis, this observation provides a spectral resolution of $\delta E / E \sim 0.43(0.93 \mathrm{keV} / E)^{0.5}$. The PSPC spectral data for NGC5471B is reasonably reliable, as it is located in a relatively isolated region, $22 \mathrm{kpc}$ off the galaxy's nucleus. We find that the Raymond \& Smith thermal plasma model provides a satisfactory fit to the data $\left(\chi^{2} /\right.$ d.o.f. $\left.=6.2 / 11\right)$, giving the plasma temperature as $0.29(0.22-0.45)$ and the absorbing-gas column density as $1.8(0.3-6.1) \times 10^{20} \mathrm{~cm}^{-2}$; the parameter intervals are all at the $90 \%$ confidence. The solar metal abundance is assumed for X-ray-absorbing gas, which affects only the absolute value of the column density. The normalization of the model is

$$
K \equiv\left[10^{-14} /\left(4 \pi D^{2}\right)\right] \int n_{e}^{2} d V \sim 8.2 \times 10^{-5} \mathrm{~cm}^{-5}
$$

where $n_{e}$ is the electron density of the plasma, and the integration is over the entire volume of the remnant. The metal abundance $\zeta$ of the X-ray-emitting plasma is assumed as $13 \%$ solar, as inferred from accurate measurements of oxygen abundance in NGC5447 (Torres-Peimbert, Peimbert, \& Fierro, 1989). The temperature of X-ray-emitting plasma indicates an expanding shock velocity of $\sim 5.0 \times 10^{2} \mathrm{~km} \mathrm{~s}^{-1}$, consistent with the lower limit inferred from the optical kinematics. This, together with the relatively small optical size of NGC5471B, suggests the remnant is in the Sedov-Taylor phase.

We can thus further estimate physical parameters of NGC5471B, using the Sedov solution:

$$
R=(20 \mathrm{pc})\left(2 E_{52} t_{4}^{2} / n_{0}\right)^{1 / 5},
$$

where $R, E_{52}, t_{4}$, and $n_{0}$ are the radius (in units of pc), blastwave energy $\left(10^{52}\right.$ ergs), age $\left(10^{4} \mathrm{yrs}\right)$, and preshock density $\left(\mathrm{cm}^{-3}\right)$ of the remnant. The shocked gas temperature can be expressed as

$$
k T \sim\left(1.8 \times 10^{-3} \mathrm{keV}\right)\left(R / t_{4}\right)^{2},
$$

and the postshock-to-preshock density ratio is $n_{e} / n_{0}=4$. From (1), (2), and (3), we obtain

$$
\begin{gathered}
n_{0} \sim 2 \mathrm{~cm}^{-3}(R / 30 \mathrm{pc})^{-3 / 2}\left(K / 8 \times 10^{-5} \mathrm{~cm}^{-5}\right)^{1 / 2}, \\
E \sim\left(3 \times 10^{52} \mathrm{ergs}\right)(R / 30 \mathrm{pc})^{3 / 2}\left(K / 8 \times 10^{-5} \mathrm{~cm}^{-5}\right)^{1 / 2}(k T / 0.3 \mathrm{keV}),
\end{gathered}
$$

and

$$
t \sim\left(2 \times 10^{4} \mathrm{yrs}\right)(k T / 0.3 \mathrm{keV})^{-1 / 2}(R / 30 \mathrm{pc}) .
$$

This age is smaller than the time for the pressure-driven snowplow (PDS) phase to start

(Cioffi, McKee, \& Bertschinger 1988), $t_{P D S} \sim\left(5 \times 10^{4}\right.$ yrs $) n_{0}^{4 / 7} E_{52}^{3 / 14} \zeta_{0.13}^{-5 / 14}$, consistent 
with our assumption of the Sedov-Taylor phase. We further estimate the total thermal energy as $\sim 10^{52}$ ergs and the gas cooling rate as $\sim 10^{39} \mathrm{ergs} \mathrm{s}^{-1}$. The inferred cooling time is $\sim 3 \times 10^{5} \mathrm{yrs}$, and thus the remnant is apparently not in a radiative phase. The estimate of $E$, in particular, is not sensitive to the uncertainty in the spectral parameters, because $K$ anti-correlates with $k T$ (Fig. 2). Thus, NGC5471B most likely originates in a super-energetic explosion - a hypernova.

Similarly, we can combine the optical and X-ray results to constrain the physical properties of MF83. Its large radius and relatively low shock velocity suggest that this remnant has evolved into the PDS phase, which starts at the radius $R_{P D S}=(32 \mathrm{pc}) E_{52}^{2 / 7} n_{0}^{-3 / 7} \zeta_{0.3}^{-1 / 7}$ and velocity $v_{P D S}=\left(376 \mathrm{~km} \mathrm{~s}^{-1}\right) E_{52}^{1 / 14} n_{0}^{1 / 7} \zeta_{0.3}^{3 / 14} . \zeta=0.3$ is assumed to be the same as the abundance measured for the nearby GHC NGC5461 (Torres-Peimbert, Peimbert, \& Fierro, 1989), and the parameter values to be inferred are not sensitive to this assumption. The PDS evolution (Cioffi, McKee, \& Bertschinger 1988) follows

$$
R=R_{P D S}\left[(4 / 3)\left(t / t_{P D S}\right)-1 / 3\right]^{3 / 10}
$$

and

$$
v_{e}=v_{P D S}\left[(4 / 3)\left(t / t_{P D S}\right)-1 / 3\right]^{-7 / 10} .
$$

From these two equations and the values of $R$ and $v_{e}$ (Table 1), we get $E_{52} \sim 13 n_{0}^{1.2}$. If $n_{0} \gtrsim 0.1 \mathrm{~cm}^{-2}$, MF83 is then a hypernova remnant (HNR) as well. We may estimate $n_{0}$ from the X-ray data on MF83. However, the remnant is not bright enough in X-ray and is in a relatively crowded region; no reliable $\mathrm{X}$-ray spectral constraint is yet available. Nevertheless, if the X-ray spectrum (e.g., the plasma temperature) is not too much different (a factor of $\lesssim 3$ ) from that of NGC5471B, the X-ray luminosity of MF83 suggests a total thermal energy $E_{T} \sim 6 \times 10^{52}$ ergs. Taking into account the adiabatic energy loss during the PDS phase, $E \sim E_{T}\left(R / R_{P D S}\right)^{2}$, we obtain $n_{0}=(32 / R)^{46 / 15}\left(376 / v_{e}\right)^{11 / 5} E_{T, 52}^{31 / 30} \sim 2 \mathrm{~cm}^{-3}$; thus, $t \sim 1 \times 10^{6}$ yrs and $E \sim 3 \times 10^{53}$ ergs.

While the above calculations are based on a simple SNR evolution model, the consideration of potential complications may significantly alter the estimates of the parameters. For example, if an explosion occurs in a pre-existing low-density (stellar wind) bubble, the actual blastwave energy can then be a factor of a few lower than our estimate (e.g., Hughes et al. 1998). The expanding velocity of MF86 is also uncertain. But we do not expect that such complications would affect our results qualitatively. The strong X-ray emission as well as the unusual optical and radio characteristics of NGC5471B and MF83 all suggest that they originate in super-energetic explosions. 


\section{Hypernova Remnants and GRBs}

The detection of HNRs in nearby galaxies may have important implications for our understanding of GRBs. The recent discovery of afterglows strongly suggests that GRBs represent the most extreme high-energy events we have witnessed in the universe. In the extreme case (GRB 990123), the inferred isotropic energy release ( $z 3 \times 10^{54}$ ergs) exceeds the rest mass of a neutron star (Kulkarni et al. 1999), although the beaming of the emission may substantially reduce the energy requirement (e.g., Mészáros, Rees, \& Wijers 1998 and references therein). Furthermore, such an event may contain 10 times more energy in outflowing non-relativistic matter (Loeb \& Perna 1998), which is not observable during a GRB and its afterglow. Most plausible models of GRBs involve either the coalescence of two compact objects - two neutron stars or a neutron star and black hole - or collapses of massive stars and/or their mergers with compact companions (Paczyński 1998; Fryer \& Woosley 1998). The later scenarios have become particularly attractive, because of the evidence that GRB/afterglow events appear close to star-forming regions. In all these models, an event leads to the formation of a black hole and provides an extractable energy of $\sim 10^{54}$ ergs (Mészáros, Rees, \& Wijers 1998). This naturally raises the hope to find in nearby galaxies relics of similar events as they must have interacted with the interstellar medium (ISM). Indeed, it has been suggested recently by Efremov, Elmegreen, \& Hodge (1998) and by Loeb \& Perna (1998) that some of H I supershells or holes in the ISM may represent such relics. As demonstrated in these papers, however, it is very difficult to distinguish the GRB scenario from other more conventional interpretations of the supershells: the combined action of stellar winds and SN of OB associations or impacts of high-velocity clouds. OB associations, for example, can be hard to detect within H I supershells with typical ages $\gtrsim 10^{7}$ yrs.

In contrast, a relatively young HNR, or a young GRB relic, in a nearby galaxy can be identified relatively easily and can be studied in great details at multi-wavelengths. Except for its substantially greater blastwave energy, an HNR resembles an SNR and should have such distinct signatures as nonthermal radio continuum, high $[\mathrm{S} \mathrm{II}] / \mathrm{H} \alpha$ ratio, large shock velocity, and strong soft X-ray emission, as observed from NGC5471B and MF83. These signatures are not expected for a supershell powered even by a young OB association. Energetically, one can argue that NGC5471B or MF83 might be a result of multiple SN explosions. But this would require an explosion rate of $\gtrsim 1$ per $10^{4}$ yrs, or a mean mechanical energy input of $z 10^{40} \mathrm{ergs} \mathrm{s}^{-1}$, within the boundaries of each remnant. Such concerted energy input could only occur in very massive OB associations, more massive than 30 Dor in the Large Magellanic Cloud (e.g., Wang 1999). To be consistent with the sizes of NGC5471B and MF83, the massive OB associations would also have to be very young (less than several times $10^{6} \mathrm{yrs}$ ). Such young OB associations, however, 
would produce luminous HII nebulae, similar to the 30 Dor nebula with predominantly thermal characteristics, which do not fit the observations of either NGC5471B or MF83. One could further recognize the associations in optical images. But, there is no evidence for their presence within both NGC5471B and MF83 (Chu 1999, privately communications). Similarly, one can distinguish young HNRs from small ring-like or half-shell features which might be produced by recent impacts of high-velocity clouds. At an expected velocity comparable to the galactic disk rotation, the impact could hardly heat gas to a temperature of a few times $10^{6} \mathrm{~K}$, which should be typical for young HNRs. In conclusion, one should be able to identify relics of GRBs if they are present as young HNRs in nearby galaxies.

Could NGC5471B and MF83 be the relics of GRBs statistically? From our sample of 2 HNRs with their ages within $\sim 1 \times 10^{6}$ yrs, we may infer a rate of order 2 hypernovae per $10^{6}$ yrs in M101. Within the obvious uncertainties, this rate agrees with theoretical estimates from stellar evolution models for binary neutron star mergers or induced neutron star collapses in binary systems for an $L^{*}$ galaxy (e.g. Qin et al. 1998), and may also be comparable to estimated hypernova rates, which depend largely on the history of star formation (e.g., Fryer \& Woosley 1998). Observationally, GRBs appear to be about $10^{4}-10^{6}$ rarer than SN (Wijers et al. 1998). Assuming an SN rate of 1 per 50 yrs (MF) in M101, one then expects 0.02-2 GRBs per $10^{6} \mathrm{yrs}$. Our inferred rate is at the upper end of this range. This may be explained by two important factors. First, the SN rate in M101 is of great uncertainty; the MF survey covers only part of the galaxy and a substantial number of SNRs as expected in GHCs are not detected (MF). The true SN rate may be considerably higher in M101. Second, only a fraction of hypernovae are likely observable as GRBs at cosmological distances, presumably due to a moderate beaming effect of $\gamma$-ray emission as expected in GRB models. Therefore, the detection of the 2 remnants in M101 is reasonably consistent with the GRB statistics.

Clearly, our detection of the 2 apparently young HNRs in M101 represents only the first step to firmly establish their identity. No matter what this identity might be, however, these remarkable remnants must originate in very energetic events. Other nearby galaxies may also contain similar remnants. In particular, there is a population of persistent X-ray sources with luminosities greater than the Eddington limit for a $\sim 1.6 M_{\odot}$ neutron star. Such superluminous sources tend to be associated with galaxies of active massive star formation, and are absent in members of the Local Group (e.g., Wang et al. 1999). It is conceivable that some of these sources, as in M101, may arise in HNRs. The upcoming $A X A F$ and $X M M$ will be able to spatially resolve such remnants and to obtain high spectral resolution data over the broad 0.1-10 keV range. The X-ray data, combined with detailed observations of the remnants in other wavelength bands, will provide important constraints on the rate, blastwave energy distribution, and progenitor type of the events. 
Most excitingly, such a study may open up a new avenue to explore the origin and physics of GRBs.

The author is indebted to Y.-H. Chu for her sharing insights and valuable information about the remnants during the course of this work, which is supported partly by NASA LTSA grant NAG5-6413. He is also grateful to A. Loeb, S. Oey, and B. Paczyński for stimulating discussions on GRBs as well as on hypernovae and their remnants.

\section{REFERENCES}

Chen, C.-H. R., \& Chu, Y.-H. 1999, BAAS, 193, 7405

Chu, Y.-H., \& Kennicutt, R. C. Jr. 1986, ApJ, 311, 85

Cioffi, D. F., McKee, C. F., \& Bertschinger, E. 1988, ApJ, 334, 252

Efremov, Y. N., Elmegreen, B. G., \& Hodge, P. W. 1998, ApJL, 501, 163

Fryer, C., \& Woosley, S. 1998, ApJL, 502, 9

Hughes, J, P., Hayashi, I., Koyama, K. 1998, ApJ, 505, 732

Israel, F. P., Goss, W. M., \& Allen, R. J. 1975, A\&A, 40, 421

Kulkarni, S., et al. 1999, Nature, submitted

Loeb, A., \& Perna, R. 1998, ApJL, 503, 35

Matonick, D. M., \& Fesen, R. A. 1997, ApJS, 112, 49 (MF)

Mészáros, P., Rees, M. J., \& Wijers, R. A. M. J. 1999, New Astronomy, in press

Paczyński, B. 1998, ApJL, 494, 45

Qin, B., Wu, X.-P., Chu, M.-C., Fang, L.-Z., \& Hu, J.-Y. 1998, ApJL, 494, 57

Skillman, E. D. 1985, ApJ, 290, 449

Stetson, P. B., et al. 1998, ApJ, 508, 491

Torres-Peimbert, S., Peimbert, M., \& Fierro, J. 1989, ApJ, 345, 1

Wang, Q. D. 1999, ApJL, 510, 139

Wang, Q. D., Immler, S., \& Pietsch, W. 1999, ApJ, submitted

Wijers, R.A.M.J., et al. 1998, MNRAS, 294, L13

Yang, H., Skillman, E. D., \& Sramek, R. A. 1994, AJ, 107, 651 
Table 1: X-ray-emitting Remnants Candidates in M101

\begin{tabular}{|c|c|c|c|c|c|c|c|c|c|c|c|c|}
\hline $\begin{array}{l}\text { SNR } \\
\text { Name }\end{array}$ & $\begin{array}{c}\text { R.A. } \\
\left(\begin{array}{lll}h & \text { m }\end{array}\right)\end{array}$ & 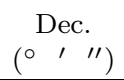 & $\begin{array}{c}R_{g} \\
(\mathrm{kpc})\end{array}$ & $\begin{array}{c}\Delta_{x-o} \\
\left({ }^{\prime \prime}\right)\end{array}$ & $\begin{array}{c}r_{e r r}(1 \sigma) \\
\left({ }^{\prime \prime}\right)\end{array}$ & $\begin{array}{c}\mathrm{L}(\mathrm{H} \alpha) \\
\left(\operatorname{ergs~s}^{-1}\right)\end{array}$ & $\begin{array}{l}{[\mathrm{SII}]} \\
/ \mathrm{H} \alpha\end{array}$ & $\begin{array}{r}\operatorname{Size}^{a} \\
(\mathrm{pc})\end{array}$ & $\begin{array}{c}v_{e} \\
\left(\mathrm{~km} \mathrm{~s}^{-1}\right)\end{array}$ & $\begin{array}{l}\text { Optical } \\
\text { Shape }\end{array}$ & $\begin{array}{l}\text { RHRI } \\
\text { Source }\end{array}$ & $\begin{array}{c}L_{x} \\
\left(\operatorname{ergs~s}^{-1}\right)\end{array}$ \\
\hline MF37 & 14304.1 & 542736 & 14 & 3.3 & 3.0 & $4.3 \mathrm{E} 37$ & 0.72 & - & - & - & H19 & $6.9 \mathrm{E} 38$ \\
\hline MF54 & 14320.7 & 541942 & 3.6 & 6.2 & 3.1 & $3.5 \mathrm{E} 37$ & 0.52 & 27 & - & filled & $\mathrm{H} 29$ & $1.4 \mathrm{E} 38$ \\
\hline MF57 & 14324.2 & 541944 & 4.2 & 4.0 & 3.1 & $1.1 \mathrm{E} 37$ & 0.47 & 40 & - & filled & H30 & 1.1E38 \\
\hline MF83 & 14335.9 & 541924 & 7.7 & 1.8 & 3.1 & 1.7E38 & 0.76 & 267 & $\sim 86$ & shell & H36 & $1.2 \mathrm{E} 38$ \\
\hline NGC5471B & 14429.1 & 542353 & 24 & 3.1 & 3.4 & $4.0 \mathrm{E} 38^{b}$ & $0.51^{b}$ & $60^{b}$ & $\gtrsim 175^{c}$ & shell & H49 & $3.0 \mathrm{E} 38$ \\
\hline
\end{tabular}

${ }^{a}$ The sizes of MF SNRs are recalculated, using the M101 distance of 7.2 Mpc instead of 5.4 Mpc assumed by MF97.

${ }^{b}$ from Chen \& Chu (1999).

${ }^{c}$ from Chu \& Kennicutt (1986). 
Fig. 1.- RHRI intensity contour map overlaid on an optical image of M101 from Palomar Sky Survey. The map is adaptively smoothed with a Gaussian of size adjusted to achieve a constant signal-to-noise ratio of $\sim 6$. The contours are at 1.1, 1.6, 2.4, 5.3, and $12 \times 10^{-3}$ counts s$^{-1} \operatorname{arcmin}^{-2}$; the lowest level is about $3 \sigma$ above the local X-ray background. The locations of the X-ray-emitting remnant candidates (Table 1) are marked.

Fig. 2. - 68\%, 90\%, and 99\% confidence contours of the spectral parameters: $k T$ vs. normalization of the Raymond \& Smith thermal plasma fit to the X-ray spectral data of NGC5471B. The cross marks the best-fit parameter values. 
This figure "sfig1.gif" is available in "gif" format from: http://arxiv.org/ps/astro-ph/9903246v1 
This figure "sfig2.gif" is available in "gif" format from: http://arxiv.org/ps/astro-ph/9903246v1 